\title{
Gall Stones - Prevention with a Plant-Based Diet
}

\author{
Stewart D Rose and Amanda J Strombom* \\ Plant-Based Diets in Medicine, USA
}

Submission: December 12, 2019; Published: January 10, 2020

*Corresponding author: Amanda Strombom, Plant-Based Diets in Medicine, 12819 SE 38th St, \#427, Bellevue, WA 98006, USA

Abstract

A plant-based diet has been shown to reduce the risk of cholesterol gall stones. This risk reduction is supported by the fact that vegetarians, and especially vegans, have a much lower prevalence of risk factors for cholesterol gallstones such as reduced risk of obesity, insulin resistance, Type 2 Diabetes and Crohn's disease. Pregnant women are particularly susceptible to cholesterol gallstones due to the impact of weight gain, insulin resistance, leptin increases and hormonal changes. Since treatment for extreme hypercholesterolemia with medication during pregnancy is risky, a plant-based diet is indicated.

A plant-based diet excludes foods shown to increase the risk of cholesterol gallstones such as meat, poultry, fish along with the heme iron, saturated fat and dietary cholesterol which have been shown to increase cholesterol in gallstone disease patients. At the same time, a plant-based diet includes fruits, vegetables, whole grains, legumes rich with fiber along with mono and polyunsaturated oils, which have been shown to reduce the risk of cholesterol gallstones.

Keywords: Gall Stones; Cholesterol gall stones; Obesity; Insulin resistance; Crohn's disease; Hypercholesterolemia; Polyunsaturated oils; Cholelithiasis; Cholecystitis; Pancreatitis; Gastrointestinal disease; Ascorbic acid; Gallbladder disease; Hepatitis C virus infection

\section{Introduction}

Gallstone disease constitutes a major health burden that has increased more than $20 \%$ over the last 3 decades [1-3]. The prevalence of gall stones (cholelithiasis) is about 10 to 15 percent of the population of the U.S., or well over 25 million people [1,4]. Nearly 1 million new cases of gallstone disease are diagnosed every year and approximately one quarter of these require treatment.

The burden of cholelithiasis and its complications, such as cholecystitis, pancreatitis, and cholangitis, are major public health problems. With an estimated 1.8 million ambulatory care visits each year, gallstone disease is a leading cause for hospital admissions related to gastrointestinal disease [5]. A 2006 study reported that more than 700,000 cholecystectomies were performed in the United States at a cost of $\$ 6.5$ billion dollars annually [6]. Cholecystectomy is now the most common elective abdominal surgery performed in the United States [7-9]. These numbers are likely an underestimate because laparoscopic cholecystectomy is often performed as a day procedure and thus not captured by hospital statistics that require overnight admission. Also not captured in the statistics is the pain of biliary colic that many patients experience.

\section{Risk Factors}

Several studies, comparing those with gallstones versus those without, have shown that cholesterol gallstone formation is multifactorial. Some risk factors, such as ethnicity, genetics, advancing age, and female gender (including pregnancy) cannot be modified, whereas diet, BMI, Type 2 diabetes, and Crohn's Disease (plus excess alcohol consumption and cigarette smoking) can be modified [4]. With regards to diet, studies have reported that the risk of gallstones was positively associated with intake of meat, excess calories, fat and saturated fat, but negatively associated with intake of vegetables and fiber [10]. Nutritional exposure to the western diet, i.e. increased intake of fat, refined carbohydrates and a decrease in fiber content, is a major risk factor for the development of gallstones [10]. In Japan, postwar westernization has provided an example for the interplay between environment and disease. Since the late 1940's, the prevalence of gallstones in Tokyo has more than doubled. Moreover, there has been a change from pigment to cholesterol gallstones and the sex ratio has changed in favor of females [11]. This increase in gallstone incidence was associated with an increased fat intake and a decreased fiber content of the diet, and consequently was attributed to the westernization of the Japanese diet [12]. 
When assessing the prevalence of gallstone disease, one must take into account that many people have gallstones but are asymptomatic. Approximately 20\% become symptomatic after 10 years of follow up [13]. Comparing non-vegetarians to vegetarians, a study of both symptomatic and asymptomatic sonographically-confirmed cholelithiasis cases, found that the prevalence of gallstones was 1.9 time higher in non-vegetarians than in vegetarians [14]. While most studies show a decreased risk of gall stones among vegetarians, one did not although this should be regarded with caution, since it runs counter to the greater weight of evidence [15]. The lower risk that vegetarians seem to experience begs the question of which dietary factors are operational. A study of vegetarians examining this question, found that although the prevalence of gallstone disease was less than half that of non-vegetarians, there was no difference in the intake of macronutrients between the study and control groups. In this cross-sectional study, the prevalence of gallbladder disease (asymptomatic gallstones or history of cholecystectomy) was significantly lower in vegetarians than omnivores $(12 \%$ versus 25\%) [16].

Heme iron, which is present in red meat, fish, and poultry, is highly bioavailable. In one large cohort study, a higher intake of heme iron was associated with a higher risk of gallstone disease, with a dose-response relation that was not accounted for by other potential risk factors [17]. Another study, which looked not at total fat, but saturated fat versus mono- and poly-unsaturated fat, found that after adjustment for age and other potential risk factors, compared with men in the lowest quintile of dietary intake of long-chain saturated fats, the relative risk of gallstone disease for men in the highest quintile was $24 \%$ higher and the risk of cholecystectomy $41 \%$ higher than for omnivores [18]. Medium-chain saturated fatty acid and short-chain saturated fatty acid intake were unrelated to the risk. Short-chain saturated fats are found in the colon as the result of microbial action on dietary fiber, and medium-chain saturated fats occur naturally in plant foods, whereas long-chain fatty acids are primarily found in animal fats. Complementing the above study was one that showed that polyunsaturated fats decrease the risk of gallstone formation by $18 \%$ for men in the highest quintile of dietary intake of cis unsaturated fats, compared with men in the lowest quintile [19]. Consumption of vegetable protein is associated with a reduced risk of cholecystectomy [20]. In addition, adherence to a diet rich in fruit, vegetables, legumes, and olive oil has been associated with a reduction in cholecystectomy risk in women [21-23].

Fiber decreases the transit time thereby lowering the risk of gall bladder disease and cholecystectomy in women [24]. In another study, women with the highest insoluble fiber intake had a $17 \%$ lowered risk of gallbladder surgery [25]. A study on the benefit of consuming nuts found that men consuming 5 or more ounces of tree nuts per week had a $30 \%$ lower risk of gallstone disease [26]. Both obesity and insulin resistance are associated with gallbladder disease [27,28]. BMI is a strong risk factor for gall bladder disease [4]. Vegetarians and vegans have significantly lower BMI's on average. A study of American vegetarians and vegans found that that vegetarians had a mean BMI of 25.7 and vegans a mean BMI of 23.6 [29]. A European study found the average BMI of vegetarians and vegans to be 23.3 and 22.4 respectively for men and 22.8 and 21.8 for women [30]. A study of German vegans found an average BMI of 22.3 [31].

There is an increased risk of gallbladder disease among Type 2 diabetes patients [27]. Vegetarians, and even more so vegans, have less insulin resistance, and have a greatly reduced risk of T2DM [32]. This and their lower risk of obesity contribute to their lower risk of gallstones. The prevalence of gallstones in patients with Crohn disease ranges from $13 \%$ to $34 \%$ in different studies, accounting for a total of about 700 patients [33]. However, the risk of Crohn's disease has been found to be reduced by $70 \%$ in female and $80 \%$ in male young people following a nearly vegetarian diet [34]. As a result of this, vegetarians appear to have a lower risk of cholesterol gallstones because of their higher intake of fruits, vegetables, nuts, fiber and plant protein and also their lower BMI, risk of type 2 diabetes and Crohn's disease.

\section{Pathogenesis}

Cholesterol gallstone pathogenesis must be briefly considered to facilitate the presentation of risk factors. Cholesterol gallstones constitute more than $80 \%$ of stones in the Western world. Cholesterol gallstones, composed predominantly of cholesterol crystals, result from abnormalities in cholesterol metabolism [12]. Four types of abnormalities have been considered to be responsible for cholesterol gallstone formation. The first and essential requirement is bile supersaturation in cholesterol. The second abnormality is enhanced nucleation of cholesterol crystals. Mucin and its congeners, the major proteins, act as matrix molecules to hold cholesterol crystal aggregates together to form a stone [12].

There also must be sufficient time for nucleation to occur, for crystals to form and grow to microliths, and for microliths to aggregate to form gallstones, hence gallbladder stasis is a contributing factor to gallstone formation. During overnight fasting, the gallbladder does not empty so that hours of storage occur in all individuals [12]. Intestinal hypomotility has been recently recognized as a fourth primary factor in cholesterol lithogenesis. Having a longer exposure to intestinal micro-organisms, primary bile salts are in greater proportion deconjugated and dehydroxylated to more hydrophobic secondary bile salts. An increased proportion of the secondary bile acid deoxycholate, a potent down-regulator of the rate-limiting enzyme for bile acid biosynthesis, enhances cholesterol hypersecretion into bile [12].

Excessive cholesterol secretion could result from defective conversion of cholesterol to bile acids, due to a low or relatively low activity of cholesterol $7 \alpha$ hydroxylase, the rate limiting 
enzyme for bile acid biosynthesis and cholesterol elimination [35]. Ascorbic acid found in many fruits and vegetables reduces lithogenic risk in adults in that it influences $\alpha$ hydroxylase activity in the bile [10].

Cholesterol supersaturation, the essential requirement for cholesterol gallstone formation, might occur via excessive cholesterol biosynthesis (increased 3-hydroxy-3-methylglutaryl (HMG) coenzyme A (CoA) reductase activity) [35]. Hepatic metabolism of cholesterol in gall stone patients is hypothesized to be different than in normal patients [36]. In gallstone disease, a patient's cholesterol and bile acid homeostasis is significantly altered. As a patient's dietary cholesterol increases, biliary cholesterol secretion increases and bile acid synthesis and pool decrease. These are changes associated with cholesterol gallstone formation $[35,37]$.

\section{Gallstone Disease in Pregnancy}

Epidemiological and clinical studies have found that gallstone prevalence is twice as high in women as in men at all ages in every population studied. In pregnancy in particular, the incidence of cholesterol gallstones is increased by strong risk factors including obesity, serum leptin and extreme hypercholesterolemia. The incidence rates of biliary sludge (a precursor to gallstones) and gallstones are up to $30 \%$ and $12 \%$, respectively, during pregnancy and postpartum, and gallbladder disease is the most common non obstetrical cause of maternal hospitalization in the first year postpartum [38,39]. Between $1 \%$ and $3 \%$ of pregnant women undergo cholecystectomy due to clinical symptoms or complications within the first year postpartum.

During the period of a normal, healthy pregnancy, the body undergoes substantial hormonal, immunological, and metabolic changes [40-44]. Some of these alterations become risk factors contributing to the formation of cholesterol gallstones in pregnant women, including weight gain, insulin resistance, and an altered gut microbiota [40,45-51]. Insulin resistance is a risk factor for incident gallbladder sludge and stones during pregnancy, even after adjustment for body mass index [52-54]. Increased estrogen levels during pregnancy induce significant metabolic changes in the hepatobiliary system, including the formation of cholesterol supersaturated bile and sluggish gallbladder motility, two factors enhancing cholelithogenesis. During pregnancy, bile becomes lithogenic because of a significant increase in estrogen levels, which lead to hepatic cholesterol hypersecretion and biliary lithogenicity. In addition, increased progesterone concentrations impair gallbladder motility function, with the resulting increase in fasting gallbladder volume and bile stasis. Such abnormalities greatly promote the formation of biliary sludge and gallstones. Because plasma concentrations of female sex hormones, especially estrogen, increase linearly with the duration of gestation, the risk of gallstone formation becomes higher in the third trimester of pregnancy [40].
Serum leptin is a strong predictor of incident gallbladder disease in pregnant women [55]. However, a plant-based diet has been shown to lower leptin levels [56]. Pre pregnancy obesity is a strong risk factors for pregnancy-associated gallbladder disease [55]. Those following a plant-based diet have, on average, a lower BMI thus reducing their risk of pre pregnancy obesity which is a strong risk factor for gall stone disease [32]. Prescribing a prenatal plant-based diet for prophylaxis is therefore indicated.

Prevention of gallstone disease is especially important in pregnancy when cholecystectomy is more problematic and medication can carry risks. At present extreme hypercholesterolemia is not treated during pregnancy, partly due to the absence of established normal parameters for pregnancy, as well as clinicians' uncertainty as to the significance of elevated levels for a limited time period. HMG CoA-reductase inhibitors (statins), which are the most commonly used drugs to treat high cholesterol outside of pregnancy, are contraindicated [57].

With established pregnancy-specific reference values, extreme maternal hypercholesterolemia could be identified and monitored. Due to recognized adverse effects of very high cholesterol on the pregnancy and the fetus, intervention with a cholesterol-lowering diet may be necessary and prove beneficial [57]. A plant-based diet has been shown to lower the risk of obesity, reduce leptin and eliminate cholesterol from the diet, thus reducing serum cholesterol during pregnancy without the risk that drug treatment may entail [58]. The American Academy of Nutrition and Dietetics position statement on plant-based diets confirms their safety during pregnancy and lactation and that they may confer additional health advantages [59].

\section{Gallstone Disease in Vegetarians}

While the risk of cholelithiasis is lower in vegetarians, a study looked at the risk factors that remained among vegetarians themselves. Risk factors useful for predicting cholelithiasis in vegetarians are age and total bilirubin level in men, and age, BMI, and alcohol consumption in women. Just as interesting is some indication of those factors which weren't risk factors in this study. Many previously identified risk factors for the general population do not seem to apply to vegetarians. The study revealed that diabetes mellitus, coronary artery disease, cerebral vascular accident, chronic renal failure, hepatitis $\mathrm{C}$ virus infection, and lipid abnormalities were not associated with cholelithiasis in male and female vegetarians. This finding may be explained by the protective effect of vegetarian diets, according to the authors [36].

Counseling patients who are already vegetarian should focus on modifiable risk factors, such as BMI and alcohol consumption, to reduce their risk still further. Some research has focused on determining which dietary factors account for the lower risk vegetarians have of gallstone disease. One study found that men consuming 5 or more ounces of tree nuts per week had a 
significantly lower risk of gallstone disease [26]. In another study, those consuming the most fruits and vegetables were $21 \%$ less likely than those with the lowest intake to require cholecystectomy [23].

\section{Discussion}

While a plant-based diet cannot treat gallstones that have already formed, considering the scale of gallstone disease, the possibility of preventing about half the cases is very significant. It is particularly valuable as a way of reducing the risk of gallstones during pregnancy, when pharmaceutical methods are not possible. The plant-based diet can also prevent and treat common comorbidities such as type 2 diabetes, obesity and coronary artery disease. It has no adverse reactions and has no contraindications.

\section{References}

1. Shaffer EA (2005) Epidemiology and risk factors for gallstone disease: has the paradigm changed in the 21st century? Curr Gastroenterol Rep $7(2): 132-140$.

2. Sandler RS, Everhart JE, Donowitz M, Adams E, Cronin K, et al. (2002) The burden of selected digestive diseases in the United States. Gastroenterology 122(5): 1500-1511.

3. Everhart JE, Ruhl CE (2009) Burden of digestive diseases in the United States part I: overall and upper gastrointestinal diseases. Gastroenterology 136(2): 376-386.

4. Stinton LM, Shaffer EA (2012) Epidemiology of gallbladder disease: Cholelithiasis and cancer. Gut Liver 6(2): 172-187.

5. Shaheen N, Hansen R, Morgan D, Gangarosa L, Ringel Y, et al. (2006) The burden of gastrointestinal and liver diseases. Am J Gastroenterol 101(9): 2128-2138.

6. Shaffer EA (2006) Epidemiology of gallbladder stone disease. Best Pract Res Clin Gastroenterol 20(6): 981-996.

7. Everhart JE, Khare M, Hill M, Maurer KR (1999) Prevalence and ethnic differences in gallbladder disease in the United States. Gastroenterology 117(3): 632-639.

8. Kang J, Ellis C, Majeed A, Hoare J, Tinto A, et al. (2003) Gallstones--an increasing problem: a study of hospital admissions in England between 1989/1990 and 1999/2000. Aliment Pharmacol Ther 17(4): 561-569.

9. Russo M, Wei J, Thiny M, Gangarosa L, Brown A, et al. (2004) Digestive and liver diseases statistics. Gastroenterology 126(5): 1448-1453.

10. Elkhateeb Y, Alghannam T, Alkhamali S (2019) Gallbladder Stone Disease and its Relation to Dietary Intake. Acta Scientific Gastrointestinal Disorders 2(3): 17-20.

11. Kameda H, Ishihara F, Shibata K, Tsukie E (1984) Clinical and nutritional study on gallstone disease in Japan. Jpn J Med 23(2): 109-113.

12. Acalovschi M (2001) Cholesterol gallstones: from epidemiology to prevention. Postgrad Med J 77(906): 221-229.

13. Attili A, DeS antis A, Capri R, Repice A, Maselli S (1995) The natural history of gallstones: the GREPCO experience. The GREPCO Group. Hepatology 21(3): 655-660.

14. Pixley F, Wilson D, McPherson K, Mann J (!985) Effect of vegetarianism on development of gall stones in women. Br Med J 291(6487): 11-12.

15. Mc Connell T, Appleby P, Key T (2017) Vegetarian diet as a risk factor for symptomatic gallstone disease. Eur J Clinil Nutr 71(6):731-735.
16. Pixley F, Mann J (1988) Dietary factors in the aetiology of gall stones: a case control study. Gut 29(11): 1511-1515.j

17. Tsai C, Leitzmann M, Willett W, Giovannucci E (2007) Heme and nonheme iron consumption and risk of gallstone disease in men. Am J Clin Nutr 85(2): 518-522.

18. Tsai C, Leitzmann M, Willett W, Giovannucci E (2008) Long-chain saturated fatty acids consumption and risk of gallstone disease among men. Ann Surg 247(1): 95-103.

19. Tsai C, Leitzmann M, Willett W, Giovannucci E (2004) The effect of longterm intake of cis unsaturated fats on the risk for gallstone disease in men: A prospective cohort study. Ann Intern Med 141(7): 514-522.

20. Tsai C, Leitzmann M, Willett W, Giovannucci E (2004) Dietary protein and the risk of cholecystectomy in a cohort of US women: the Nurses' Health Study. Am J Epidemiol 160(1): 11-18.

21. Barré A, Gusto G, Cadeau C, Carbonnel F, Boutron Ruault M (2017) Diet and Risk of Cholecystectomy: A Prospective Study Based on the French E3N Cohort. Am J Gastroenterol 112(9): 1448-1456.

22. Nordenvall C, Oskarsson V, Wolk A (2018) Fruit and vegetable consumption and risk of cholecystectomy: a prospective cohort study of women and men. Eur J Nutr 57(1):75-81.

23. Tsai C, Leitzmann M, Willett W, Giovannucci E (2006) Fruit and vegetable consumption and risk of cholecystectomy in women. Am J Med 119(9): 760-767.

24. Tsai C, Leitzmann M, Willett W, Giovannucci E (2004) Long-term intake of dietary fiber and decreased risk of cholecystectomy in women. Am J Gastroenterol 99(7): 1364-1370.

25. Hillemeier C (1995) An overview of the effects of dietary fiber on gastrointestinal transit. Pediatrics 96(5 Pt 2): 997-999.

26. Tsai C, Leitzmann M, Hu F, Willett W, Giovannucci E (2004) A prospective cohort study of nut consumption and the risk of gallstone disease in men. Am J Epidemiol 160(10): 961-968.

27. Aune D, Vattena L (2016) Diabetes mellitus and the risk of gallbladder disease: A systematic review and meta-analysis of prospective studies. J Diabetes Complications 30(2): 368-373.

28. Aune D, Norat T, Vatten L (2015) Body mass index, abdominal fatness and the risk of gallbladder disease. Eur J Epidemiol 30(9): 1009-1019.

29. Tonstad S, Butler T, Yan R, Fraser G (2009) Type of Vegetarian Diet, Body Weight, and Prevalence of Type 2 Diabetes. Diabetes Care 32(5): 791-796.

30. Bradbury K, Crowe F, Appleby P, Schmidt J, Travis R, et.al. (2014) Serum concentrations of cholesterol, apolipoprotein A-I, and apolipoprotein B in a total of 1694 meat-eaters, fish-eaters, vegetarians, and vegans. Eur J Clin Nutr 68(2): 178-183.

31. Waldmann A, Koschizke J, Leitzmann C, Hahn A. (2005) German vegan study: diet, life-style factors, and cardiovascular risk profile. Annuls of Nutrition and Metabolism 49(6): 366-72.

32. Strombom A, Rose S (2017) The prevention and treatment of Type II Diabetes Mellitus with a plant-based diet. Endocrin Metab Int J 5(5): 342-354.

33. Fraquelli M, Losco A, Visentin S, Cesana B, Pometta R, et al. (2001) Gallstone disease and related risk factors in patients with Crohn disease: analysis of 330 consecutive cases. Arch Intern Med 161(18): 2201-2204.

34. Rose S, Strombom A (2018) Crohn's disease prevention and treatment with a plant-based diet. Adv Res Gastroentero Hepatol 9(1): 555-753. 
35. Kern F (1994) Effects of dietary cholesterol on cholesterol and bile acid homeostasis in patients with cholesterol gallstones. J Clin Invest 93(3): 1186-1194.

36. Chen Y, Chiou C, Lin M, Lin C (2014) The prevalence and risk factors for gallstone disease in Taiwanese vegetarians. PLoS One 9(12): e115145.

37. Greenberger N, Paumgartner G (2008) Diseases of the Gallbladder and Bile Ducts. In: Fauci AS, Braunwald E, Kasper DL, (Eds.), Harrison's Principles of Internal Medicine. (17 ${ }^{\text {th }}$ edn.), McGraw-Hill Professional.

38. Ko C (2006) Risk factors for gallstone-related hospitalization during pregnancy and the postpartum. Am J Gastroenterol 101(10): 22632268.

39. Lydon Rochelle M, Holt V, Martin D, Easterling T (2000) Association between method of delivery and maternal rehospitalization. JAMA 283(18): 2411-2416.

40. De Bari O, Wang T, Liu M, Paik C, Portincasa P, et al. (2014) Cholesterol cholelithiasis in pregnant women: pathogenesis, prevention and treatment. Ann Hepatol 13(6): 728-745.

41. Newbern D, Freemark M (2011) Placental hormones and the control of maternal metabolism and fetal growth. Curr Opin Endocrinol Diabetes Obes 18(6): 409-416

42. Murphy V, Smith R, Giles W, Clifton V (2006) Endocrine regulation of human fetal growth: the role of the mother, placenta, and fetus. Endocr Rev 27(2): 141-169.

43. Barbour L, Mc Curdy C, Hernandez T, Kirwan J, Catalano P, et al. (2007) Cellular mechanisms for insulin resistance in normal pregnancy and gestational diabetes. Diabetes Care 30(Suppl 2): S112-119.

44. Lain K, Catalano P (2007) Metabolic changes in pregnancy. Clin Obstet Gynecol 50(4): 938-948.

45. Zavalza Gómez A, Anaya Prado R, Rincón Sánchez A, Mora Martínez J (2008) Adipokines and insulin resistance during pregnancy. Diabetes Res Clin Pract 80(1): 8-15.

46. Mor G, Cardenas I (2011) The Immune System in Pregnancy: A Unique Complexity. Am J Reprod Immunol 63(6): 425-433.

47. Jamieson D, Theiler R, Rasmussen S (2006) Emerging infections and pregnancy. Emerg Infect Dis 12(11): 1638-1643.
48. Koren O, Goodrich J, Cullender T, Spor A, Laitinen K, et al. (2012) Host remodeling of the gut microbiome and metabolic changes during pregnancy. Cell 150(3): 470-480.

49. Ko C, Beresford S, Schulte S, Lee S (2008) Insulin resistance and incident gallbladder disease in pregnancy. Clin Gastroenterol Hepatol 6(1): 76-81.

50. Collado M, Isolauri E, Laitinen K, Salminen S (2008) Distinct composition of gut microbiota during pregnancy in overweight and normal-weight women. Am J Clin Nutr 88(4): 894-899.

51. DiCianni G, Miccol R, Volpe iL, Lencioni C, DelPrato S (2003) Intermediate metabolism in normal pregnancy and in gestational diabetes. Diabetes Metab Res Rev 19(4): 259-270.

52. Gielkens H, Lama W, Coenraad M, Frölich M, van Oostayen J, et al. (1998) Effect of insulin on basal and cholecystokinin-stimulated gallbladder motility in humans. J Hepatol 28(4): 595-602.

53. Haffner S, Diehl A, Mitchell B, Stern M, Hazuda H (1990) Increased prevalence of clinical gallbladder disease in subjects with non-insulindependent diabetes mellitus. Am J Epidemiol 132: 327-335.

54. Ruhl C, Everhart J (2000) Association of diabetes, serum insulin, and C-peptide with gallbladder disease. Hepatology 31(2): 229-303.

55. Ko C, Beresford S, Schulte S, Matsumoto A, Lee S (2005) Incidence, natural history, and risk factors for biliary sludge and stones during pregnancy. Hepatology 41(2): 359-365.

56. Kahleova H, Matoulek M, Malinska H, Oliyarnik O, Kazdova L, et.al. (2011) Vegetarian diet improves insulin resistance and oxidative stress markers more than conventional diet in subjects with Type 2 diabetes. Diabet Med 28(5): 549-59.

57. Bartels Ä, O Donoghue K (2011) Cholesterol in pregnancy: a review of knowns and unknowns. Obstet Med 4(4): 147-151.

58. Rose S, Strombom A (2018) A comprehensive review of the prevention and treatment of heart disease with a plant-based diet. J Cardiol \& Cardiovas Ther 12(5): 555-847.

59. Melina V, Craig W, Levin S (2016) Position of the academy of nutrition and dietetics: vegetarian diets. J Acad Nutr Diet 116(12): 1970-1980.

\section{Your next submission with JuniperPublishers will reach you the below assets}

- Quality Editorial service

- Swift Peer Review

- Reprints availability

- E-prints Service

- Manuscript Podcast for convenient understanding

- Global attainment for your research

- Manuscript accessibility in different formats

( Pdf, E-pub, Full Text, audio)

- Unceasing customer service

Track the below URL for one-step submission https://juniperpublishers.com/online-submission.php 